\title{
Influence of Hypoxia in the Intervertebral Disc on the Biological Behaviors of Rat Adipose- and Nucleus Pulposus-Derived Mesenchymal Stem Cells
}

\author{
Hao Li Yiqing Tao Chengzhen Liang Bin Han Fangcai Li Gang Chen \\ Qixin Chen \\ Department of Orthopedics, Second Affiliated Hospital, Zhejiang University School of Medicine, Hangzhou, China
}

\section{Key Words}

Adipose-derived mesenchymal stem cells · Disc degeneration · Hypoxia - Intervertebral disc · Nucleus pulposus-derived mesenchymal stem cells

\begin{abstract}
Adipose-derived mesenchymal stem cells (ADMSCs) and nucleus pulposus-derived mesenchymal stem cells (NPMSCs) are two cell candidates for cell-based therapies for intervertebral disc (IVD) regeneration. However, little work has been done to determine the influence of hypoxia in the IVD on the biological behaviors of ADMSCs and NPMSCs. This study aimed to investigate the viability, proliferation and differentiation of rat ADMSCs and NPMSCs in the hypoxic environment of IVD in vitro. ADMSCs and NPMSCs isolated from 6 SD rats were cultured under normoxia $\left(20 \% \mathrm{O}_{2}\right)$ and hypoxia $\left(2 \% \mathrm{O}_{2}\right)$ mimicking the standard condition and hypoxic environment of the IVD for 14 days. Cell viability was determined by the annexin-V-FITC/propidium iodide doublestaining assay and cell proliferation was measured by MTT assay. The expression of hypoxia-inducible factor- $1 a$, glucose transporter (GLUT)-1, GLUT-3 and vascular endothelial
\end{abstract}

H.L. and Y.T. contributed equally to the study. growth factor-A at the mRNA level was examined by RT-PCR. In cells cultured in three-dimensional micromass and differentiation medium, aggrecan, collagen-II and Sox-9 expression at mRNA and protein levels were examined by RT-PCR and Western blot. Hypoxia inhibited the viability and proliferation of both ADMSCs and NPMSCs, but promoted the chondrocytic differentiation of ADMSCs and NPMSCs. Com-

\begin{tabular}{|c|c|}
\hline \multicolumn{2}{|c|}{ Abbreviations used in this paper } \\
\hline ADMSC & adipose-derived mesenchymal stem cell \\
\hline BMSC & bone marrow-derived mesenchymal stem cell \\
\hline $\mathrm{C}_{\mathrm{T}}$ & cycle threshold \\
\hline $3 \mathrm{D}$ & three dimensional \\
\hline DMEM-HG & Dulbecco's modified Eagle's medium-high glucose \\
\hline FCS & fetal calf serum \\
\hline GLUT & glucose transporter \\
\hline HIF & hypoxia-inducible factor \\
\hline IVD & intervertebral disc \\
\hline NP & nucleus pulposus \\
\hline NPMSC & nucleus pulposus-derived mesenchymal stem cell \\
\hline PBS & phosphate-buffered saline \\
\hline $\mathrm{PI}$ & propidium iodide \\
\hline RT-PCR & real-time quantitative polymerase chain reaction \\
\hline TBST & Tris-buffered saline containing $0.05 \%$ Tween 20 \\
\hline VEGF & vascular endothelial growth factor \\
\hline
\end{tabular}

\section{KARGER}

E-Mail karger@karger.com www.karger.com/cto
(C) 2013 S. Karger AG, Basel

1422-6405/13/1984-0266\$38.00/0 
pared to ADMSCs, NPMSCs showed greater viability, proliferation and chondrocytic differentiation under hypoxia. In conclusion, hypoxia in the IVD had a significant impact on the viability, proliferation and chondrocytic differentiation of ADMSCs and NPMSCs. NPMSCs exhibited more potent biological activity than ADMSCs in the hypoxic environment of the IVD and may represent another candidate for cell-based therapy for IVD regeneration.

(c) 2013 S. Karger AG, Basel

\section{Introduction}

Lower back pain is one of the most common health problems that cause huge medical expenses [Balague et al., 2012 ]. It is reported that this disease affects $60-80 \%$ of people at some point during their lives, with $11-12 \%$ of people being disabled by it [Steck et al., 2005; Balague et al., 2012]. Although the etiology of back pain is heterogeneous, intervertebral disc (IVD) degeneration has been proposed as a major factor that causes lower back pain [Sakai, 2008]. The IVD tissue is a specialized biomechanical complex that absorbs loads on the spinal column and provides the flexibility to the spine. The normal IVD is composed of three distinct components: the central hyperhydrated nucleus pulposus (NP), the outer annulus fibrosus, and the upper and lower cartilaginous endplates. Normal IVD matrix, including proteoglycans (predominantly aggrecan) and collagens, plays a crucial role in proper disc function [Henriksson and Brisby, 2013]. Accumulating evidence suggests that a decrease in matrix components, primarily proteoglycan and collagen-II, is an initial trigger for IVD degeneration [Alini et al., 2002; Wang et al., 2010]. At present, treatments for diseases resulting from IVD degeneration, both conservative and invasive, are based on symptomatic relief, but they do not reverse the degeneration process or repair the degenerated IVD tissue [Sakai, 2008; Blanco et al., 2010]. Therefore, biological approaches, including cellbased therapy, have been widely investigated for the treatment of IVD degeneration.

Stem cells have the ability to self-renew, proliferate and differentiate into several cell types and hold great potential for cell therapy programs, including bone and cartilage repair [Wang et al., 2013; Xue et al., 2013]. Currently, stem cells have attracted much attention as a cell source for cell-based IVD regeneration [Sakai, 2008]. Many studies indicated that stem cell transplantation is a promising approach to delay or reverse IVD degeneration [Ganey et al., 2009; Yang et al., 2009]. Furthermore, some of the implanted stem cells can differentiate into

Influence of Hypoxia in the IVD on ADMSCs and NPMSCs chondrocyte-like NP cells, which increase the amount of matrix in the IVD [Zhang et al., 2005; Sakai et al., 2006; Le Maitre et al., 2009; Yang et al., 2009]. In a mouse annular puncture model of IVD degeneration, Yang et al. [2009] found that bone marrow-derived mesenchymal stem cell (BMSC) transplantation could arrest the progressive IVD degeneration with significant NP regeneration. The results of in situ hybridization and immunohistochemistry revealed that BMSCs underwent chondrocytic differentiation in the degenerated IVD. In addition, Le Maitre et al. [2009] investigated the survival and phenotype of human BMSCs injected into bovine NP tissue explants. After a 4-week culture, BMSCs differentiated spontaneously into chondrocyte-like NP cells which expressed Sox-9, aggrecan and collagen-II.

Adipose-derived mesenchymal stem cells (ADMSCs) are somatic stem cells that are abundant in fat tissue with the virtue of easy isolation and comparative abundance [Chun et al., 2012]. In vitro studies indicated that ADMSCs could differentiate into NP-like cells, which express aggrecan and collagen-II [Lu et al., 2008; Tapp et al., 2008]. Additionally, ADMSC-based transplantation can arrest IVD degeneration or even partially regenerate IVD in many animal models [Ganey et al., 2009; Chun et al., 2012]. Recently, several studies have demonstrated that the IVD itself contains stem cells [Blanco et al., 2010; Liu et al., 2011; Mark Erwin et al., 2013]. Blanco et al. [2010] identified NP-derived mesenchymal stem cells (NPMSCs) from human NP tissues and found that NPMSCs fulfilled nearly all morphological, immunophenotypic and differentiation criteria described by the International Society of Cell Therapy for mesenchymal stromal cells. These findings suggest that NPMSCs may be an endogenous stem cell that could be applied to repair degenerated IVD.

In adulthood, the IVD is the largest avascular tissue in the human body [Ciapetti et al., 2012]. Blood vessels just infiltrate the superficial region of the cartilaginous endplates and the outer third of the annulus fibrosus [Rudert and Tillmann, 1993]. In the avascular IVD, essential nutrients such as oxygen and glucose diffuse from the capillaries across the cartilaginous endplate and through the dense disc matrix to the cells. There are steep gradients in oxygen concentration across the IVD with oxygen levels ranging from 1 to $5 \%$ in vivo [Mwale et al., 2011]. During degeneration, structural changes with vascular depletion, endplate calcification and disc size increase make oxygen diffusion harder and the oxygen concentration in the IVD becomes even lower [Roughley, 2004; Galbusera et al., 2013]. 
Table 1. RT-PCR primers

\begin{tabular}{lll}
\hline Gene & Forward primer $\left(5^{\prime}\right.$ to $\left.3^{\prime}\right)$ & Reverse primer $\left(5^{\prime}\right.$ to $\left.3^{\prime}\right)$ \\
\hline Nanog & GGAACGCTGCTCCGCTCCATA & GCTTCCAAATTCGCCTCCAAATCAC \\
Sox-2 & CCCCCGTGGTTACCTCTTCC & GTGCTGGGCCATGTGCAGTC \\
Rex-1 & CACCCATGTCCGCATCCACA & CTCGACTTTCAGCATTTCTTCCCT \\
Cct-4 & CTGCAGAAGGAGCTAGAGCAGTTTG & CTCGAAGCGGCAGATGGTTGT \\
CD90 & CCGCAGAGGCTTGTTGCCAGTA & CACAGCAGTGCGGTGTCTTTCT \\
CD73 & TGTCCCGAGGACAGAGGGTGA & CTGGATGGGCAAGTTGGTGTTATTC \\
CD45 & GATCCCCTCCTCAGAAATGTCAAA & CCAACAATCCCCACAACCTCAC \\
CD34 & GAGCATTCCACGGGTATTCAGCA & GTGGACCCTGCGTCTCCATTTATT \\
HIF- $\alpha$ & CGAATGCCAGATCACAGCACATTCA & GACTCCAACTGCGGCGGTTCAT \\
GLUT-1 & CAGGCTGGGCTGCCAGGTTCA & CAGCCTCCGAGGTCCTTCTCATG \\
GLUT-3 & CTCATCTCCGTTGTCCTCCAGT & CCGCTCCAATCGTGGCATAGAT \\
VEGF-A & GTCACCACCACACCACCATCGT & CTCCTCTCCCTTCATGTCAGGCT \\
Aggrecan & CTAGCTGCTTAGCAGGGATAACG & GATGACCCGCAGAGTCACAAAG \\
Collagen-II & CTGGTGGAGCAGCAAGAGC & GTGGACAGTAGACGGAGGAAAG \\
Sox-9 & AGGAAGCTGGCAGACCAGTACC & GGGTCTCTTCTCGCTCTCGTTCA \\
$18 S$ & GAATTCCCAGTAAGTGCGGGTCATA & CGAGGGCCTCACTAAACCATC \\
\hline
\end{tabular}

While ADMSCs and NPMSCs may offer ideal cell sources for cell-based degenerated IVD regeneration, the biological behaviors of these stem cells in the hypoxic environment of the IVD remain largely uninvestigated. Therefore, the goal of the present study was to investigate the viability, proliferation and differentiation of ADMSCs and NPMSCs in the hypoxic environment of the IVD.

\section{Materials and Methods}

\section{Cells Isolation, Culture and Characterization}

All procedures involving animals were approved by the Ethics Committee of the Zhejiang University. Six male SD rats (4 months old, 250-300 g) were included in this study. ADMSCs were isolated from adipose tissues surrounding the epididymis and testis. Approximately $0.5 \mathrm{~g}$ of tissue were washed with phosphate-buffered saline (PBS) and finely minced. NPMSCs were isolated from the thoracic and lumbar IVD tissue of the rats. The IVD samples were cleaned of extraneous ligaments and NP tissue was separated from the annulus fibrosus tissue under a sterilized dissecting microscope.

Then, the minced adipose or NP tissue was digested with $0.2 \%$ collagenase-II (Sigma-Aldrich, St. Louis, Mo., USA) in Dulbecco's modified Eagle's medium-high glucose (DMEM-HG; Gibco-BRL, Grand Island, N.Y., USA) at $37^{\circ} \mathrm{C}$ for $40-60 \mathrm{~min}$ in a water bath shaker (200 rpm), respectively. The collagenase was inactivated by the addition of DMEM-HG supplemented with $10 \%$ fetal calf serum (FCS; Gibco). The ADMSC- or NPMSC-containing cell suspensions were centrifuged at $1,500 \mathrm{rpm}$ for $10 \mathrm{~min}$ at room temperature, respectively. The liberated cells and partially digested tissue were plated in $25-\mathrm{cm}^{2}$ cell culture flasks (Costar Corning, Corning, N.Y., USA) and cultured in DMEM-HG supplemented with 10\% FCS, penicillin (50 U/ml; Gibco) and streptomycin (50 $\mu \mathrm{g} /$ $\mathrm{ml}$; Gibco; standard medium) at $37^{\circ} \mathrm{C}$ with $5 \% \mathrm{CO}_{2}$. For ADMSC isolation, cultures were washed with PBS after $48 \mathrm{~h}$ to remove unattached cells and partially digested tissue. For NPMSCs isolation, cultures were washed with PBS after $96 \mathrm{~h}$. Then, cells were fed by complete replacement of the standard medium twice a week.

ADMSCs and NPMSCs were expanded up to passage 2 and were characterized by stemness gene expression and multiple differentiation. The expression levels of stemness genes (Nanog, Sox2 , Rex-1 and Oct-4) and mesenchymal stem cell surface marker genes (CD105, CD90, CD73, CD45 and CD34) were assessed by real-time quantitative polymerase chain reaction (RT-PCR). NP cells of the rat served as negative controls. Briefly, total RNA was extracted from cells using TRIzol (Invitrogen, Carlsbad, Calif., USA). RT-PCR was performed using an iCycler system (Bio-Rad Laboratories, Hercules, Calif., USA) and iQ ${ }^{\mathrm{TM}}$ SYBR Green super mix PCR kit (Bio-Rad). 18S-RNA was used as internal control. The primers used are shown in table 1 . The cycle threshold $\left(\mathrm{C}_{\mathrm{T}}\right)$ value for each gene was corrected using the mean $\mathrm{C}_{\mathrm{T}}$ value. RT-PCR data were quantified using the $\Delta \mathrm{C}_{\mathrm{T}}$ method with the formula: $\mathrm{n}=100$ $\times 2^{-\left(\Delta \mathrm{C}_{\mathrm{T}} \text { targeted gene }-\Delta \mathrm{C}_{\mathrm{T}} 18 \mathrm{~S}-\mathrm{RNA}\right)}$. Isolated ADMSCs and NPMSCs were also characterized by osteogenic and adipogenic differentiation. Osteogenic differentiation using an osteogenesis kit (Cyagen Biosciences, Guangzhou, China) was confirmed by positive alizarin red (Sigma) staining of mineralized matrix after 21 days of culture. Adipogenic differentiation using an adipogenesis kit (Cyagen Biosciences) was confirmed by oil red O (Sigma) staining of lipid droplets after 14 days of culture.

\section{Cell Culture}

ADMSCs and NPMSCs were cultured in $25-\mathrm{cm}^{2}$ plastic culture flasks for cell viability, in 24-well plates for the cell proliferation assay and in three-dimensional (3D) micromass for differentiation analysis. For normoxic conditions, culture dishes were placed at $37^{\circ} \mathrm{C}$ with $5 \% \mathrm{CO}_{2}$. For hypoxic conditions, culture dishes were 
placed in a hypoxia chamber (Biospherix, Redfield, N.Y., USA) with a mixture of $2 \% \mathrm{O}_{2}, 5 \% \mathrm{CO}_{2}$ and $93 \% \mathrm{~N}_{2}$.

For differentiation analysis, $1.0 \times 10^{6}$ ADMSCs or NPMSCs in $3 \mathrm{ml}$ of standard medium were centrifuged at 1,000 rpm for $5 \mathrm{~min}$ in a 15 -ml polypropylene conical centrifuge tube to form a cell micromass. Firstly, ADMSCs or NPMSCs were collected at the bottom of the $15-\mathrm{ml}$ centrifuge tube. Then, the tube was transferred to an incubator at $37^{\circ} \mathrm{C}$ with $5 \% \mathrm{CO}_{2}$ and the cap of the tube was loosened to allow air exchange. After $48 \mathrm{~h}$ of culture, cells aggregated spontaneously and formed a spherical micromass. Finally, the spherical micromasses were maintained in DMEM-HG supplemented with $5 \mu \mathrm{g} / \mathrm{ml}$ insulin, $5 \mu \mathrm{g} / \mathrm{ml}$ transferrin, $5 \mu \mathrm{g} / \mathrm{ml}$ selenous acid, $0.1 \mu \mathrm{M}$ dexamethasone, $0.17 \mathrm{mM}$ ascorbic acid-2-phosphate, $1 \mathrm{~mm}$ sodium pyruvate, $0.35 \mathrm{~mm}$ proline, $1.25 \mathrm{mg} / \mathrm{ml}$ bovine serum albumin and $10 \mu \mathrm{g} / \mathrm{ml}$ transforming growth factor- $\beta_{1}$ (all from Sigma). The cell micromass was cultured for 14 days and fed by complete replacement of differentiation medium twice a week.

Annexin-V-FITC/Propidium Iodide Double-Staining Assay

After 14 days of culture in $25-\mathrm{cm}^{2}$ flasks, cells were harvested, washed twice with PBS and incubated with $0.25 \mathrm{mg} / \mathrm{ml}$ annexin $\mathrm{V}$-FITC and $10 \mathrm{mg} / \mathrm{ml}$ propidium iodide (PI) for $30 \mathrm{~min}$ in the dark at room temperature. The viable, apoptotic and secondary necrotic cells were quantified by FACS analysis with a FACSCalibur cytometer (FACScan, Becton Dickinson, Mountain View, Calif., USA) equipped with CellQuest software (BD Biosciences, San Jose, Calif., USA).

\section{MTT Assay}

After 7 and 14 days of culture in 24-well plates, cell proliferation was assessed by MTT assay. Briefly, the cells were cultured in $500 \mu \mathrm{l}$ of MTT solution (250 $\mu \mathrm{g}$ in DMEM-HG) at $37^{\circ} \mathrm{C}$ for $4 \mathrm{~h}$. During this incubation period, water-insoluble formazan crystals were formed. Then, the formazan crystals were dissolved by adding $300 \mu \mathrm{l}$ of dimethyl sulfoxide. Absorbance was measured at a wavelength of $540 \mathrm{~nm}$ using a Spectra MAX microplate reader (Molecular Devices, Sunnyvale, Calif., USA).

\section{RT-PCR Analysis}

The quantification of RNA expression levels for hypoxia-inducible factor (HIF)-1 $\alpha$, glucose transporter (GLUT)-1, GLUT-3 and vascular endothelial growth factor (VEGF)-A was assessed in the cells cultured in $25-\mathrm{cm}^{2}$ flasks and standard medium. The quantification of RNA expression levels for aggrecan, collagen-II and Sox- 9 was assessed in the cells cultured in 3D micromass and differentiation medium. Briefly, total RNA was isolated from cells or cell micromass using TRIzol (Invitrogen) following the manufacturer's instructions. Six hundred nanograms of total RNA were reverse transcribed into cDNA using $1 \mu$ lof oligo(dT) primer (Invitrogen), $1 \mu \mathrm{l}$ of dNTPs and $200 \mathrm{U}$ of reverse transcriptase (Superscript II, Gibco) at $42^{\circ} \mathrm{C}$ for $60 \mathrm{~min}$. The reaction was stopped by incubation at $95^{\circ} \mathrm{C}$ for $5 \mathrm{~min}$. The quantification of mRNA levels was carried out on an iCycler system (Bio-Rad). iQ ${ }^{\mathrm{TM}}$ SYBR Green super mix PCR kit (Bio-Rad) was used for real-time monitoring of amplification (5 ng of template cDNA; 45 cycles: $95^{\circ} \mathrm{C} / 10 \mathrm{~s}$, $62^{\circ} \mathrm{C} / 25 \mathrm{~s}$ ) with appropriate primers. 18S-RNA was used as internal control. The primers used are shown in table 1 . The $\mathrm{C}_{\mathrm{T}}$ values for each gene were corrected using the mean $C_{T}$ value. RT-PCR data were quantified using the $\Delta C_{T}$ method with the formula: $\mathrm{n}=$

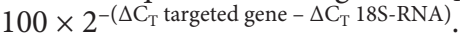

Influence of Hypoxia in the IVD on ADMSCs and NPMSCs

\section{Western Blot Analysis}

Protein expression levels for aggrecan, collagen-II and Sox-9 were assessed in the cells cultured in 3D micromass and differentiation medium. Cell micromasses were collected and, washed with ice-cold PBS and lysed in ice-cold lysis buffer (Cell Signaling Technology, Beverly, Mass., USA). The protein contents of the lysates were analyzed by BCA method, and then an amount equal to $60 \mu \mathrm{g}$ of protein was separated by $10 \%$ SDS-polyacrylamide gels and transferred to a polyvinylidene difluoride membrane. After being blocked with $5 \%$ non-fat milk in Tris-buffered saline containing $0.05 \%$ Tween 20 (TBST) for $1 \mathrm{~h}$, the membrane was incubated with the following primary antibodies: mouse monoclonal anti-aggrecan antibody (1:100; Abcam, Cambridge, Mass., USA), rabbit polyclonal anti-collagen-II antibody (1:5,000; Abcam), mouse monoclonal anti-Sox- 9 antibody (1:1,000; Santa Cruz Biotechnology, Santa Cruz, Calif., USA) and anti- $\beta$-actin antibody (1:2,000; Santa Cruz) in TBST overnight at $4^{\circ} \mathrm{C}$. The membrane was then washed three times with TBST and incubated with secondary antibody goat anti-mouse IgG/HRP (1:5,000; Pierce, Rockford, Ill., USA) or goat anti-rabbit IgG/HRP (1:5,000; Pierce) in TBST for $1 \mathrm{~h}$ at room temperature. The immunoreactive protein was detected using ECL (GE Healthcare) and BioMax film (Kodak). The relative amount of immunoreactive protein in each sample was quantitated by densitometry (AMBIS radioanalytic imaging System; Ambis, San Diego, Calif., USA).

\section{Statistical Analysis}

All data are expressed as means \pm SD. Statistical analysis was performed using SPSS 17.0 for Windows (SPSS, Chicago, Ill., USA). ADMSCs cultured under normoxia served as a control group in this study. Two-way ANOVA was used with Fisher PLSD to evaluate effects of oxygen concentration and cell type. Significance was attributed to a value of $\mathrm{p}<0.05$.

\section{Results}

\section{Characterization of ADMSCs and NPMSCs}

High expression levels of stemness genes (Nanog, Sox2, Rex-1 and Oct-4) were observed in ADMSCs and NPMSCs (fig. 1a). Both cells expressed mesenchymal stem cell surface markers CD105, CD90 and CD73, but lacked the expression of hematopoietic stem cell surface markers CD45 and CD34. ADMSCs and NPMSCs stained positive with alizarin red after culture in osteogenic media for 21 days. Red staining marked mineral deposition under a microscope (fig. 1c, f). Both cells stained positive with oil red $\mathrm{O}$ staining after culture in adipogenic media for 14 days. The lipid drop displayed red under a microscope (fig. 1d, g).

\section{Hypoxia Inhibited the Viability of ADMSCs and NPMSCs}

The viability of both ADMSCs and NPMSCs was significantly inhibited under hypoxia (fig. 2). On the 

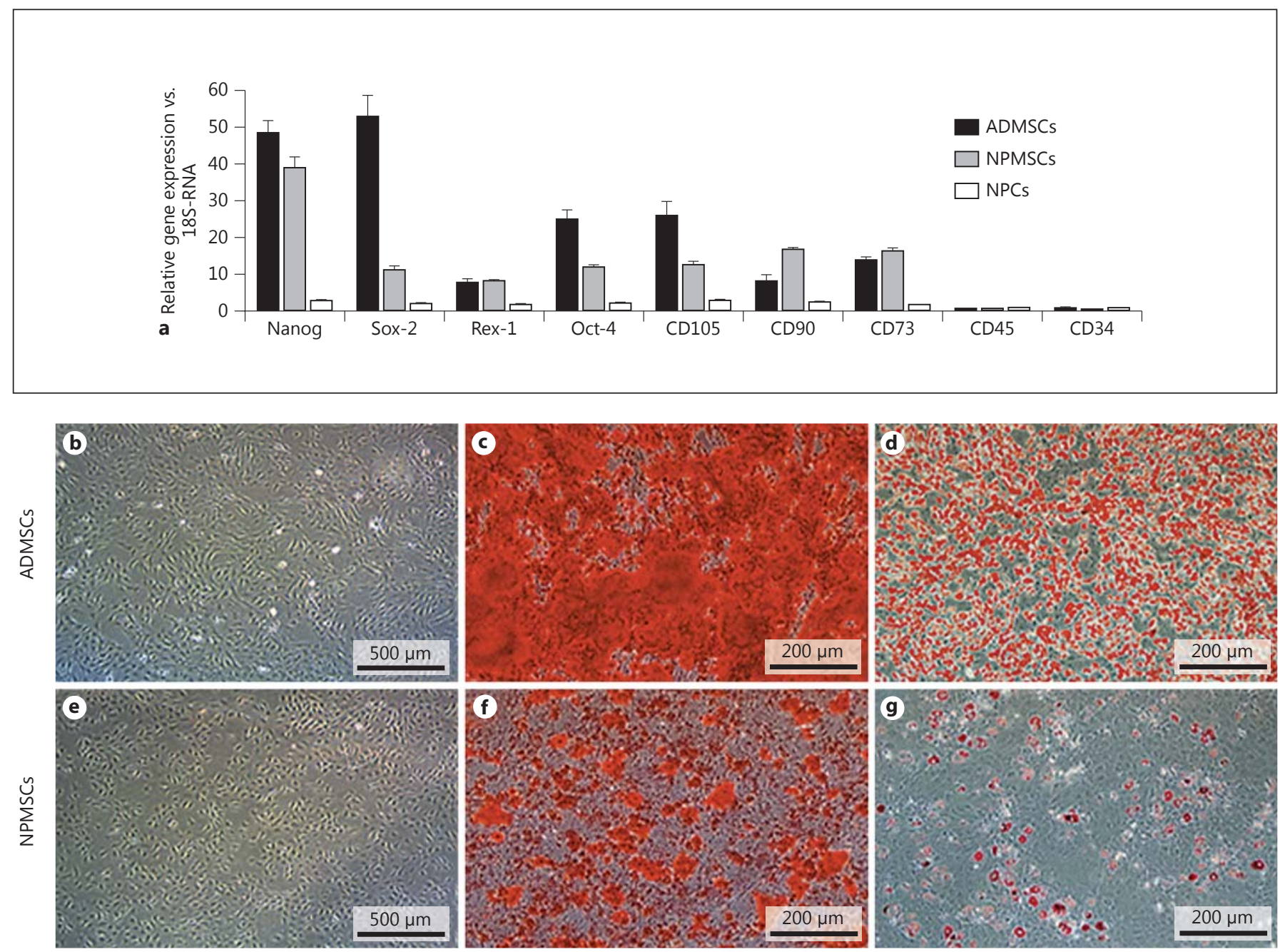

Fig. 1. Characterization of isolated ADMSCs and NPMSCs. a Stemness genes and mesenchymal stem cell surface marker gene expression in ADMSCs, NPMSCs and NP cells. High expression levels of stemness genes (Nanog, Sox-2, Rex-1 and Oct-4) were observed in ADMSCs and NPMSCs. Both ADMSCs and NPMSCs expressed mesenchymal stem cell surface markers CD105, CD90 and CD73, but lacked the expression of hematopoietic stem cell markers CD45 and CD34. Rat ADMSCs (b) and NPMSCs (e) at passage 2

basis of a 2-way ANOVA, the results showed that there was a significant influence of oxygen concentration $(\mathrm{p}<0.001)$, cell type $(\mathrm{p}<0.001)$, and an interaction between oxygen concentration and cell type $(\mathrm{p}<0.001)$ for ADMSC and NPMSC viability. Compared to normoxia, there were $6.26 \pm 2.81$-fold $(\mathrm{p}<0.001)$ and $3.00 \pm 0.91$ fold $(\mathrm{p}<0.001)$ increases in the percentages of apoptotic and necrotic cells of ADMSCs and NPMSCs under hypoxia. Under normoxia, there was no significant difference in the percentage of apoptotic and necrotic cells be- were expanded in monolayer culture. ADMSCs (c) and NPMSCs (f) were cultured in osteogenic media for 21 days and stained with alizarin red. Differentiated stem cells showed enhanced matrix calcification visualized by alizarin red staining. ADMSCs (d) and NPMSCs (g) were cultured in adipogenic media for 14 days and stained positive with oil red $\mathrm{O}$ staining. The lipid droplets in cells appeared red under a microscope.

tween ADMSCs and NPMSCs. However, the percentage of apoptotic and necrotic cells of NPMSCs was less than that of ADMSCs under hypoxia $(\mathrm{p}<0.001)$.

\section{Hypoxia Impaired the Proliferation of ADMSCs and NPMSCs}

The proliferation of both ADMSCs and NPMSCs was significantly inhibited under hypoxia (fig. 3). On the basis of a 2-way ANOVA, the result showed that there was a significant influence of oxygen concentra- 

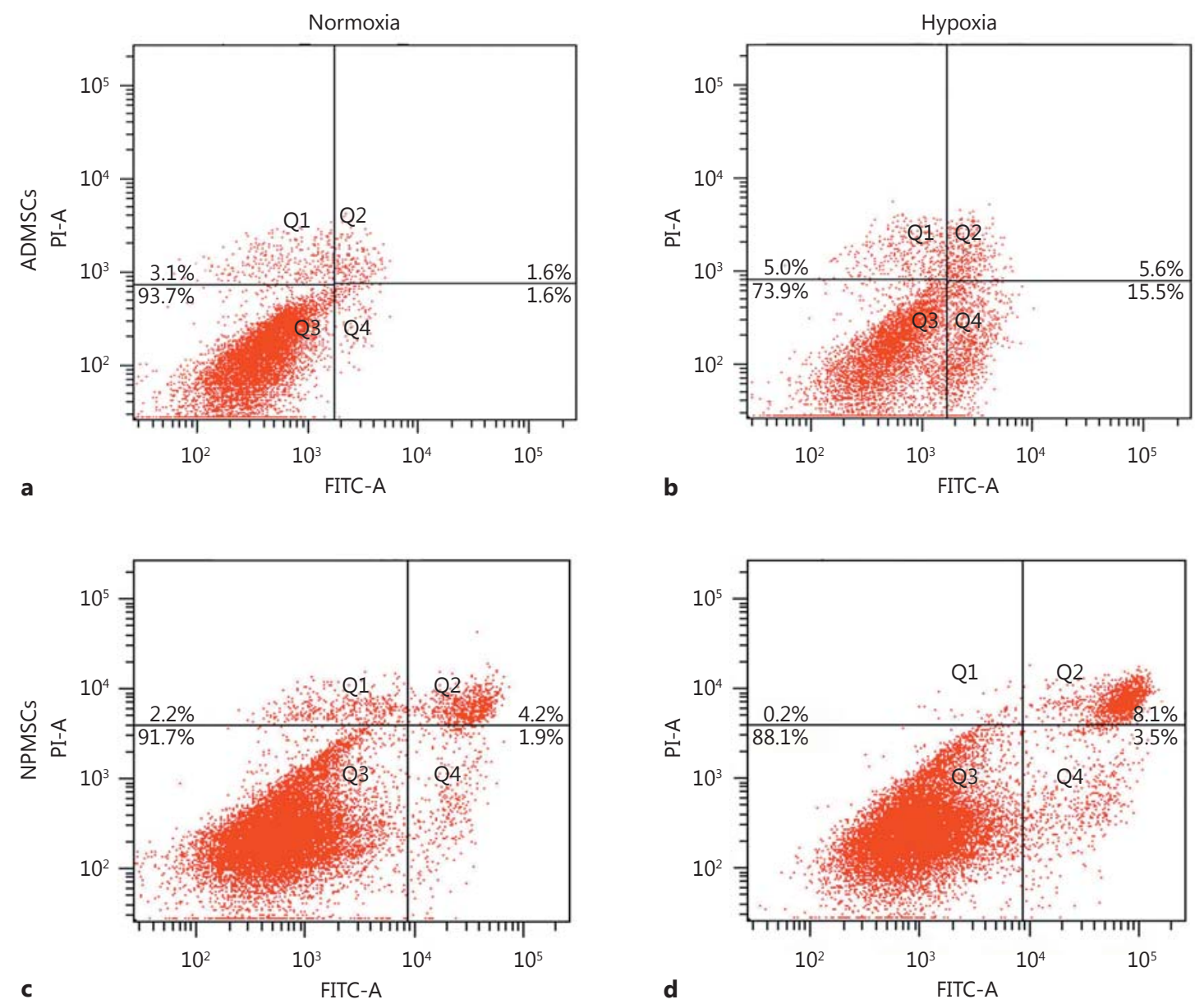

Fig. 2. Hypoxia inhibited the viability of ADMSCs and NPMSCs. The numbers of viable, apoptotic and secondary necrotic cells were determined by annexin-V-FITC/PI double staining. Percentages of apoptotic and necrotic ADMSCs from 1 SD rat under normoxia (a) and hypoxia (b). Percentages of apoptotic and necrotic NPMSCs isolated from the same rat under normoxia (c) and hypoxia (d). e Percentages of apoptotic and necrotic cells of ADMSCs and NPMSCs under hypoxia. The viability of ADMSCs and NPMSCs decreased under hypoxia compared with normoxia, and the viability of NPMSCs was greater than that of ADMSCs under hypoxia. Means \pm SE of 6 independent experiments performed in triplicate; ${ }^{* * *} \mathrm{p}<0.001$.

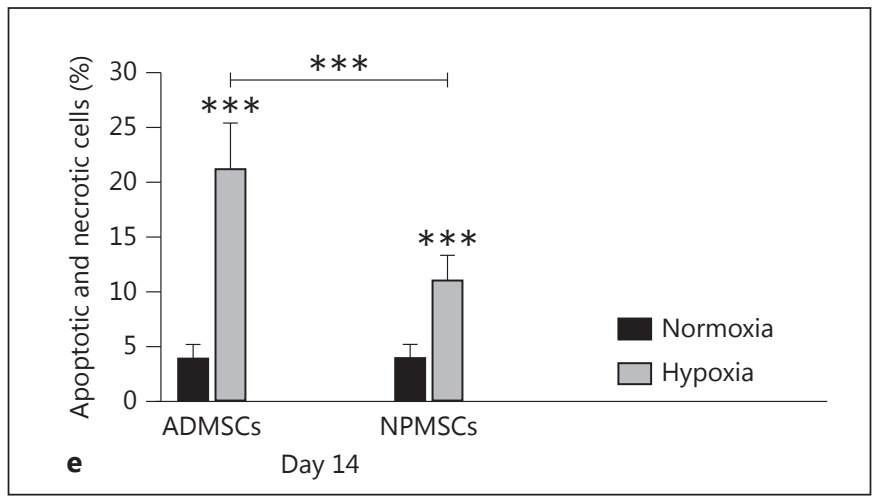

tion (both $\mathrm{p}<0.001)$ and cell type $(\mathrm{p}<0.001$ and $\mathrm{p}=$ 0.02 ), and an interaction between oxygen concentration and cell types $(\mathrm{p}=0.03$ and $\mathrm{p}=0.045)$ for ADMSC and NPMSC proliferation both on days 7 and 14. The proliferation of ADMSCs and NPMSCs was significantly

inhibited under hypoxia (ADMSCs, $55.5 \pm 6.2 \%$; NPMSCs, $75.2 \pm 4.9 \%$, both $\mathrm{p}<0.001)$ on day 7 compared to normoxia. Similar results were observed on day 14 as the proliferation was even lower under hypoxia (ADMSCs, $27.8 \pm 4.1 \%$; NPMSCs, $44.3 \pm 5.3 \%$, both $\mathrm{p}<0.001$ ). 
Fig. 3. Hypoxia impaired the proliferation of both ADMSCs and NPMSCs; the proliferation of NPMSCs was greater both on day 7 (a) and day 14 (b) under hypoxic condition. Means \pm SE of 6 independent experiments performed in triplicate; ${ }^{* *} \mathrm{p}<$ $0.01,{ }^{* * *} \mathrm{p}<0.001$.
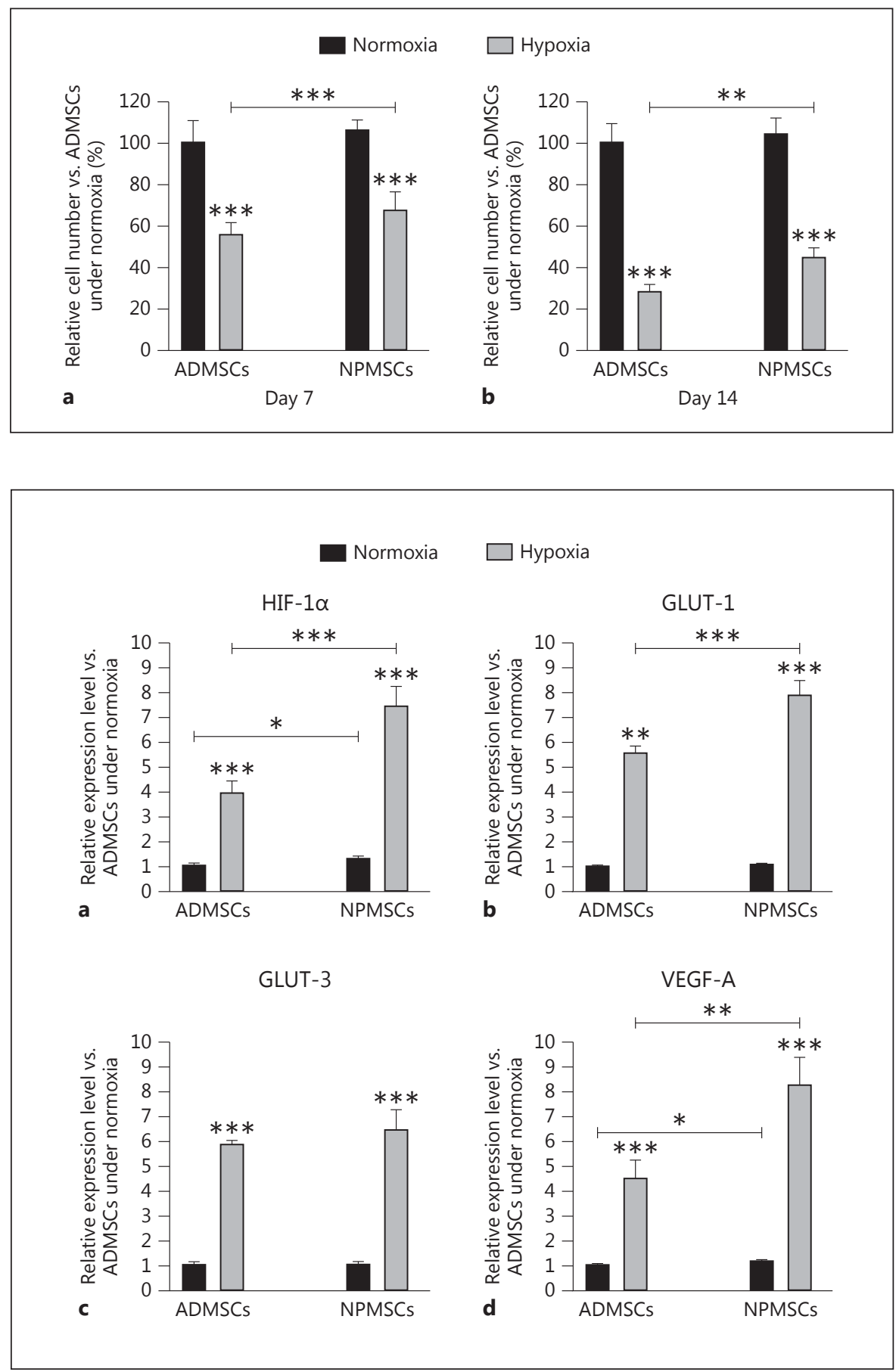

Fig. 4. Hypoxia upregulated hypoxia-responsive genes HIF-1a (a), GLUT-1 (b), GLUT-3 (c) and VEGF-A (d). The results showed that the increase in RNA expression of HIF-1a (a), GLUT-1 (b) and VEGFA (d) levels was higher in NPMSCs compared with ADMSCs under hypoxia when compared with normoxia. Means \pm SE of 6 independent experiments performed in triplicate; ${ }^{*} \mathrm{p}<0.05,{ }^{* *} \mathrm{p}<0.01,{ }^{* * *} \mathrm{p}<$ 0.001 .
Under hypoxia, some difference in the proliferation of ADMSCs and NPMSCs was observed both on days 7 ( $\mathrm{p}$ $<0.001)$ and $14(\mathrm{p}=0.001)$, with the proliferation of NPMSCs being slightly greater.

\section{Hypoxia Upregulated Hypoxia-Responsive Genes}

After 14 days of exposure to hypoxia, elevated expression of hypoxia-responsive genes, including HIF-1 $\alpha$, GLUT-1, GLUT-3 and VEGF-A, was found in both ADMSCs and NPMSCs (fig. 4). On the basis of a 2-way 


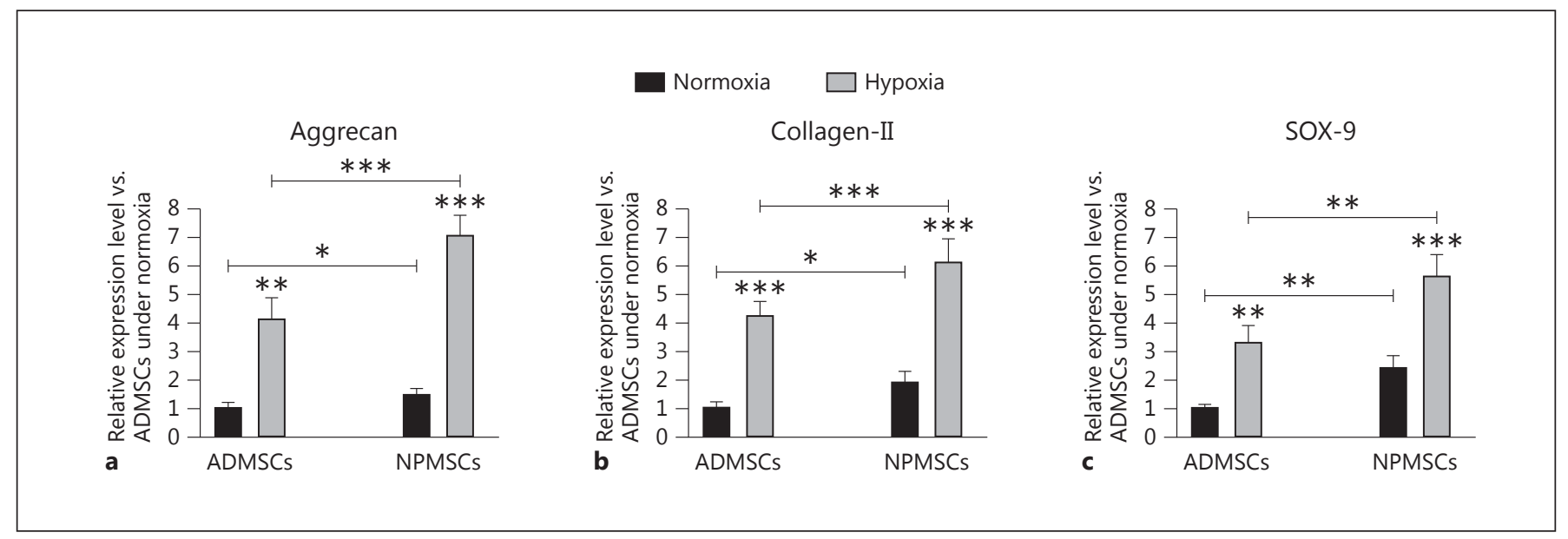

Fig. 5. Hypoxia promoted RNA expression of aggrecan, collagenII and Sox-9 in ADMSCs and NPMSCs cultured in differentiation medium. Hypoxia resulted in increased RNA expression levels of aggrecan (a), collagen-II (b) and Sox-9 (c) in both ADMSCs and NPMSCs cultured in differentiation medium for 14 days.
The increase in RNA expression of aggrecan, Sox-9 and collagenII was much higher in NPMSCs than in ADMSCs under hypoxia when compared with normoxia. Means \pm SE of 6 independent experiments performed in triplicate; ${ }^{*} \mathrm{p}<0.05$, ${ }^{* *} \mathrm{p}<0.01$, $* * * \mathrm{p}<0.001$.
ANOVA, the results indicated that there was a significant influence of oxygen concentration (both $\mathrm{p}<0.001$ ) and cell type (both $\mathrm{p}<0.001$ ), and an interaction between oxygen concentration and cell types (both $\mathrm{p}<0.001$ ) for HIF-1 $\alpha$ and VEGF-A expression; a significant influence of oxygen concentration $(\mathrm{p}<0.001)$ and cell type $(\mathrm{p}=0.003)$, and an interaction between oxygen concentration and cell types $(\mathrm{p}=0.005)$ for GLUT-1 expression, and a significant influence of oxygen concentration $(p<0.001)$ on the expression of GLUT-3. The expression levels of HIF- $1 \alpha(\mathrm{p}=$ $0.035)$ and VEGF-A ( $p=0.016)$ were higher in NPMSCs under normoxia. In addition, the expression levels of HIF$1 \alpha(\mathrm{p}<0.001)$, GLUT-1 $(\mathrm{p}<0.001)$ and VEGF-A ( $\mathrm{p}=$ 0.001 ) were higher in NPMSCs under hypoxia.

\section{Hypoxia Promoted the Chondrocytic Differentiation of ADMSCs and NPMSCs}

The expression of aggrecan, collagen-II and Sox-9 in ADMSCs and NPMSCs was measured by RT-PCR and Western blot analysis after 14 days of differentiation culture under normoxic and hypoxic conditions. The results showed that hypoxia had a significant effect on the chondrocytic differentiation of ADMSCs and NPMSCs (fig. 5, 6).

On the basis of a 2-way ANOVA, RT-PCR analysis indicated that there was a significant influence of oxygen concentration $(\mathrm{p}<0.001)$ and cell type $(\mathrm{p}<0.001)$, and an interaction between oxygen concentration and cell type

Influence of Hypoxia in the IVD on ADMSCs and NPMSCs $(\mathrm{p}<0.001)$ for aggrecan expression, and a significant influence of oxygen concentration (both $\mathrm{p}<0.001$ ) and cell type (both $\mathrm{p}<0.001$ ), and an interaction between oxygen concentration and cell type ( $\mathrm{p}=0.044 ; \mathrm{p}=0.036)$ for collagen-II and Sox-9 expression. The RNA expression levels of aggrecan (4.09 \pm 0.78 -fold, $\mathrm{p}=0.001)$, collagen-II (4.22 \pm 0.52 -fold, $\mathrm{p}<0.001)$ and Sox-9 (3.28 \pm 0.63 -fold, $\mathrm{p}=0.001)$ were elevated in ADMSCs under hypoxia compared to normoxia. Similarly, the expression of aggrecan (4.92 \pm 0.68 -fold, $\mathrm{p}<0.001)$, collagen-II $(3.34 \pm 0.64$-fold, $\mathrm{p}<0.001)$ and Sox-9 $(2.41 \pm 0.65$-fold, $\mathrm{p}<0.001)$ was increased in NPMSCs under hypoxia compared to normoxia. The RNA expression level of aggrecan $(p=0.044)$, collagen-II ( $p=0.013)$ and Sox-9 $(p=0.002)$ in NPMSCs was higher than that in ADMSCs under normoxia; the expression of aggrecan $(\mathrm{p}<0.001)$, collagen-II $(\mathrm{p}<0.001)$ and Sox-9 $(\mathrm{p}=0.001)$ in NPMSCs was also higher than that in ADMSCs under hypoxia.

On the basis of a 2-way ANOVA, Western blot analysis confirmed that there was a significant influence of oxygen concentration $(\mathrm{p}<0.001)$ and cell type $(\mathrm{p}<0.001)$, and an interaction between oxygen concentration and cell type $(\mathrm{p}<0.001)$ for aggrecan expression; a significant influence of oxygen concentration $(p<0.001)$ and cell type ( $\mathrm{p}<0.001)$, and an interaction between oxygen concentration and cell type $(\mathrm{p}=0.002)$ for collagen-II expression, and a significant influence of oxygen concentration $(\mathrm{p}<0.001)$ and cell type $(\mathrm{p}<0.001)$, and an interaction 


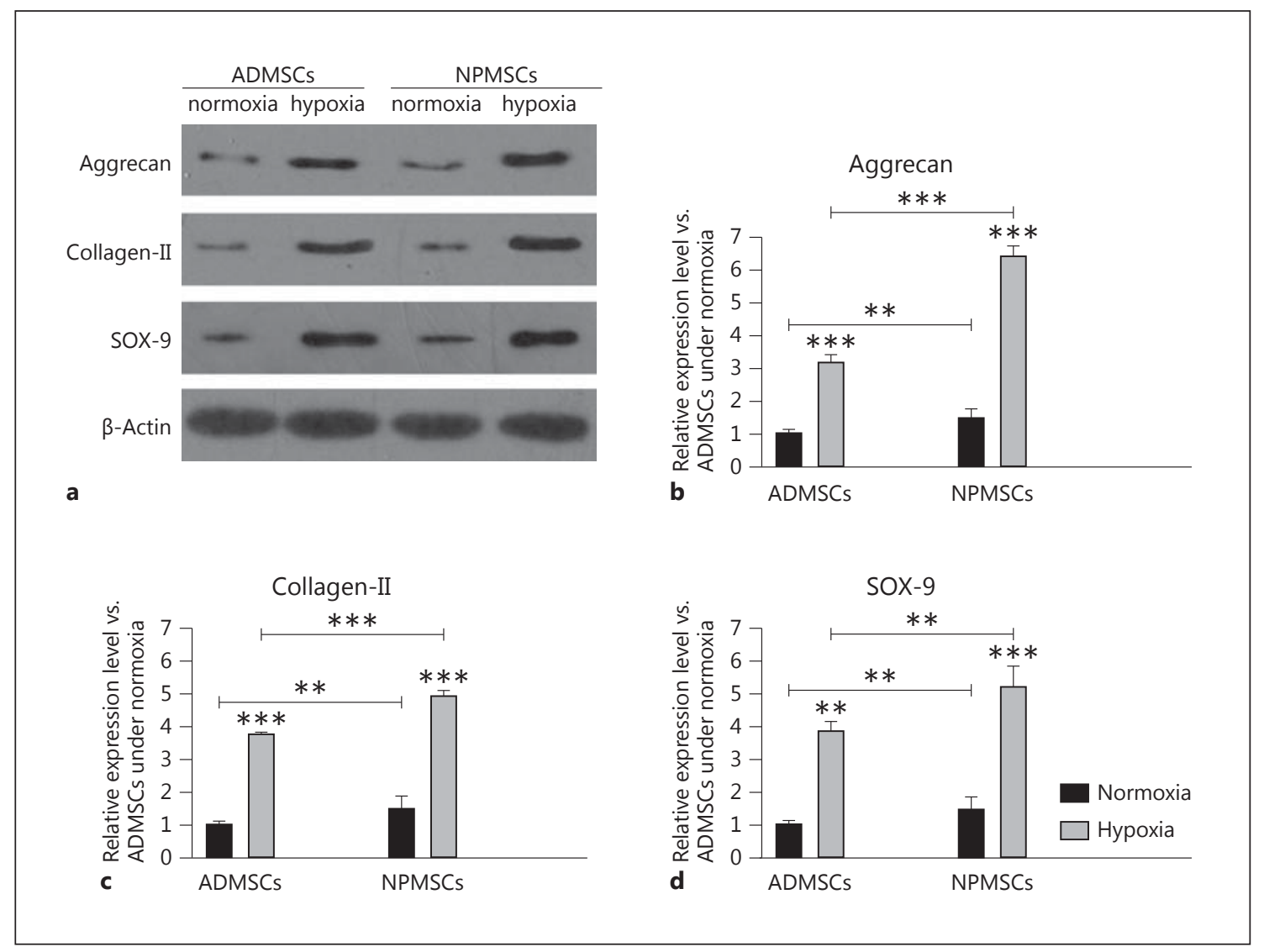

Fig. 6. Hypoxia promoted the protein expression of aggrecan, collagen-II and Sox-9 in ADMSCs and NPMSCs cultured in differentiation medium. a Western blot analysis of aggrecan, collagen-II and Sox-9 protein levels in ADMSCs and NPMSCs cultured in hypoxic differentiation medium for 14 days (representative blots

between oxygen concentration and cell type $(\mathrm{p}<0.036)$ for Sox-9 expression. The protein levels of aggrecan, collagen-II and Sox-9 were elevated in differentiated ADMSCs and NPMSCs under hypoxia compared to normoxia. In addition, the protein levels of aggrecan ( $\mathrm{p}=$ $0.009)$, collagen-II $(\mathrm{p}=0.003)$ and Sox-9 $(\mathrm{p}=0.002)$ in NPMSCs were higher than those in ADMSCs under normoxia; the expression of aggrecan $(\mathrm{p}<0.001)$, collagen-II $(\mathrm{p}<0.001)$ and Sox-9 $(\mathrm{p}=0.001)$ in NPMSCs was higher than that in ADMSCs under hypoxia.

\section{Discussion}

To date, there is increasing interest in stem cell-based therapies to reverse IVD degeneration or repair degenerated IVD. It is suggested that the maintenance, survival from 6 independent experiments with similar results). $\beta$-Actin served as a loading control. b-d Relative fold change in aggrecan (b), collagen-II (c) and Sox-9 (d) under hypoxia compared to that of ADMSCs under normoxia. Means \pm SE of 6 independent experiments performed in triplicate; ${ }^{* *} \mathrm{p}<0.01,{ }^{* * *} \mathrm{p}<0.001$. and activity of stem cells are dependent on their special environmental niche [Moore and Lemischka, 2006; Henriksson and Brisby, 2013]. The IVD exists in a hypoxic environment in vivo, thus it is necessary to understand the influence of hypoxia on the survival and activity of transplanted stem cells. In this study, we examined the effect of hypoxia in the IVD environmental niche on the biological behaviors of ADMSCs and NPMSCs. The results showed that hypoxia had a significant impact on the viability, proliferation and chondrocytic differentiation of both ADMSCs and NPMSCs; compared to ADMSCs, NPMSCs exhibited greater biological behaviors in the hypoxic environment of the IVD.

Annexin-V-FITC/PI staining showed that the viability of ADMSCs and NPMSCs was decreased under hypoxia due to cell apoptosis and secondary necrosis. The MTT assay demonstrated that the proliferation of 
ADMSCs and NPMSCs was inhibited under hypoxia, in agreement with earlier studies showing that a low oxygen concentration significantly inhibited the proliferation of ADMSCs [Wang et al., 2005; Malladi et al., 2006]. Notably, NPMSCs had greater abilities to adapt to hypoxia than ADMSCs because the viability and proliferation of NPMSCs were less affected by hypoxia. As might be expected, the reason may be the different expression levels of hypoxia-responsive genes between the two types of stem cells.

Under hypoxia, cells respond to low oxygen concentration by upregulating the expression of HIF-1 $\alpha$, a transcription factor that activates the expression of target genes involved in regulating cell survival and energy metabolism, such as GLUT-1, GLUT-3, and VEGF [Semen$\mathrm{za}, 2000,2012]$. GLUT is an integral membrane protein on the cell surface to maintain a high inward flux of glucose [Joost et al., 2002]. VEGF, a principal regulator of blood vessel formation and hematopoiesis, has been reported to improve cell survival of hematopoietic stem cells [Gerber et al., 2002] and ADMSCs [Stubbs et al., 2012]. Recent studies suggested that VEGF may serve as a survival factor for NP cells in the normal IVD [Agrawal et al., 2008; Fujita et al., 2008].

In this study, RT-PCR results showed that the expression of HIF-1 $\alpha$ and its downstream genes GLUT-1, GLUT-3 and VEGF-A in ADMSCs and NPMSCs was induced under hypoxia, and levels of HIF-1 $\alpha$, GLUT-1 and VEGF-A were higher in NPMSCs than ADMSCs under hypoxia (fig. 4). Thus, we speculate that the differences in the increased levels of HIF-1 $\alpha$, GLUT-1 and VEGF-A between ADMSCs and NPMSCs may delineate the molecular basis for their different viability and proliferation under hypoxia. Strikingly, there was no difference in the expression of GLUT-1 between ADMSCs and NPMSCs under normoxia and no difference in the expression of GLUT-3 both under normoxia and hypoxia. The mechanism is not known, and further studies are necessary to provide the explanation. Because NPMSCs had greater viability and proliferation under hypoxia, we concluded that NPMSCs may exhibit greater biological activity than ADMSCs in the hypoxic environment of IVD.

NP is a gelatinous structure composed primarily of proteoglycan and collagen. Aggrecan is a large aggregating proteoglycan which produces swelling pressure that is important for the ability of the disc to withstand compressive forces [Le Maitre et al., 2007]. The collagen fibers provide a strong framework to support the disc cells and confine the highly hydrated proteoglycan [Le Maitre et al., 2007]. Sox-9 is a key transcription factor that positively controls aggrecan and collagen-II synthesis [Li et al., 2004]. Many studies have demonstrated that stem cells could differentiate into chondrocyte-like NP cells, which express aggrecan, collagen-II and Sox-9 [Risbud et al., 2004; Lu et al., 2008; Tapp et al., 2008; Yang et al., 2009]. As the in vivo environment of the IVD is characterized by a low oxygen concentration, it is necessary to identify the effects of hypoxia on the differentiation of the transplanted stem cells. Therefore, we also investigated the influence of hypoxia on the chondrocytic differentiation of ADMSCs and NPMSCs.

Our results showed that hypoxia promoted the chondrocytic differentiation of ADMSCs and NPMSCs as it enhanced the expression of aggrecan, collagen-II and Sox-9 in these cells. The effects of hypoxia on the differentiation of both stem cells were in agreement with previous studies demonstrating that hypoxia enhanced the differentiation of BMSCs [Risbud et al., 2004]. Interestingly, the expression levels of aggrecan, collagen-II and Sox-9 in NPMSCs were much higher than those in ADMSCs both under normoxia and hypoxia. From this perspective, it was evident that NPMSCs exhibited greater differentiation ability than ADMSCs in the hypoxic environment of the IVD in vivo. This finding further strengthened the conclusion that NPMSCs exhibit greater biological activity than ADMSCs in the hypoxic environment of the IVD.

Interestingly, we observed that ADMSCs had greater osteogenic and adipogenic differentiation potential than NPMSCs (fig. 1), while NPMSCs displayed greater chondrocytic differentiation (fig. 5, 6). The precise mechanism for this inconsistent observation is unclear. One explanation could be the different culture methods because ADMSCs and NPMSCs were cultured in 2D monolayers for osteogenic and adipogenic differentiation and in $3 \mathrm{D}$ micromasses for chondrocytic differentiation. There is growing evidence that the culture system is a key factor for stem cell differentiation, and the differentiation ability of stem cells cultured in 2D and 3D is different [Hu et al., 2009; Choi et al., 2011]. It appears that 3D culture conditions play a more important role in the differentiation of NPMSCs, and future studies are needed to confirm this.

There are several limitations of the present study. First, as little is known about the signaling pathways involved in the differentiation of stem cells under the IVD hypoxic microenvironment, we did not know the precise mechanisms by which hypoxic-cultured NPMSCs exhibit greater differentiation ability than hypoxic-cultured ADMSCs. Second, the $2 \%$ oxygen concentration was chosen as the hypoxic condition in the IVD based on previous studies 
[Risbud et al., 2004; Felka et al., 2009], although the oxygen concentration is variable in the IVD. Third, the microenvironment of the IVD is harsh, which is characterized by low oxygen tension, limited nutrition, and high osmolarity and acidity. However, we only investigated the effect of hypoxia on the biological potential of ADMSCs and NPMSCs. Future studies should investigate the effects of limited nutrition, and high osmolarity and acidity of the IVD on the biological activity of these stem cells.

In summary, this study demonstrated that hypoxia had a significant effect on the viability, proliferation and chondrocytic differentiation of both ADMSCs and NPMSCs. Hypoxia inhibited the viability and proliferation of both ADMSCs and NPMSCs, but it promoted the chondrocytic differentiation of these stem cells. In addition, the study suggested that NPMSCs exhibited greater biological potential than ADMSCs in the hypoxic environment of the IVD. From this perspective, NPMSC may represent a possible candidate for cell-based therapy for IVD regeneration.

\section{Acknowledgments}

This work was supported by the grants from the National Nature Science Foundation of China (No. 81171756), the Science and Technology Planning Project of Zhejiang Province (2012C130134 and 2012C33121) and the Health Foundation of Zhejiang Province (No. 201337266).

\section{References}

Agrawal, A., S. Gajghate, H. Smith, D.G. Ander- Felka, T., R. Schafer, B. Schewe, K. Benz, W.K. son, T.J. Albert, I.M. Shapiro, M.V. Risbud (2008) Cited2 modulates hypoxia-inducible factor-dependent expression of vascular endothelial growth factor in nucleus pulposus cells of the rat intervertebral disc. Arthritis Rheum 58: 3798-3808.

Alini, M., P.J. Roughley, J. Antoniou, T. Stoll, M. Aebi (2002) A biological approach to treating disc degeneration: not for today, but maybe for tomorrow. Eur Spine J 11: S215-S220.

Balague, F., A.F. Mannion, F. Pellise, C. Cedraschi (2012) Non-specific low back pain. Lancet 379: 482-491.

Blanco, J.F., I.F. Graciani, F.M. Sanchez-Guijo, S. Muntion, P. Hernandez-Campo, C. Santamaria, S. Carrancio, M.V. Barbado, G. Cruz, S. Gutierrez-Cosio, C. Herrero, J.F. San Miguel, J.G. Brinon, M.C. del Canizo (2010) Isolation and characterization of mesenchymal stromal cells from human degenerated nucleus pulposus: comparison with bone marrow mesenchymal stromal cells from the same subjects. Spine (Phila Pa 1976) 35: 2259-2265.

Choi, E.H., H. Park, K.S. Park, B.S. Kim, I.B. Han, D.A. Shin, S.H. Lee (2011) Effect of nucleus pulposus cells having different phenotypes on chondrogenic differentiation of adipose-derived stromal cells in a coculture system using porous membranes. Tissue Eng Part A 17: 2445-2451.

Chun, H.J., Y.S. Kim, B.K. Kim, E.H. Kim, J.H. Kim, B.R. Do, S.J. Hwang, J.Y. Hwang, Y.K. Lee (2012) Transplantation of human adipose-derived stem cells in a rabbit model of traumatic degeneration of lumbar discs. World Neurosurg 78: 364-371.

-Ciapetti, G., D. Granchi, V. Devescovi, E. Leonardi, T. Greggi, M. Di Silvestre, N. Baldini (2012) Ex vivo observation of human intervertebral disc tissue and cells isolated from degenerated intervertebral discs. Eur Spine J 21: S10-S19. Aicher (2009) Hypoxia reduces the inhibitory effect of IL-1beta on chondrogenic differentiation of FCS-free expanded MSC. Osteoarthritis Cartilage 17: 1368-1376.

Fujita, N., J. Imai, T. Suzuki, M. Yamada, K. Ninomiya, K. Miyamoto, R. Iwasaki, H. Morioka, M. Matsumoto, K. Chiba, S. Watanabe, T. Suda, Y. Toyama, T. Miyamoto (2008) Vascular endothelial growth factor-A is a survival factor for nucleus pulposus cells in the intervertebral disc. Biochem Biophys Res Commun 372: 367-372.

-Galbusera, F., A. Mietsch, H. Schmidt, H.J. Wilke, C. Neidlinger-Wilke (2013) Effect of intervertebral disc degeneration on disc cell viability: a numerical investigation. Comput Methods Biomech Biomed Engin 16: 328-337.

Ganey, T., W.C. Hutton, T. Moseley, M. Hedrick, H.J. Meisel (2009) Intervertebral disc repair using adipose tissue-derived stem and regenerative cells: experiments in a canine model. Spine (Phila Pa 1976) 34: 2297-2304.

Gerber, H.P., A.K. Malik, G.P. Solar, D. Sherman, X.H. Liang, G. Meng, K. Hong, J.C. Marsters, N. Ferrara (2002) VEGF regulates haematopoietic stem cell survival by an internal autocrine loop mechanism. Nature 417: 954-958.

Henriksson, H.B., H. Brisby (2013) Development and regeneration potential of the mammalian intervertebral disc. Cells Tissues Organs 197: $1-13$.

Hu, J., K. Feng, X. Liu, P.X. Ma (2009) Chondrogenic and osteogenic differentiations of human bone marrow-derived mesenchymal stem cells on a nanofibrous scaffold with designed pore network. Biomaterials 30: 50615067.

-Joost, H.G., G.I. Bell, J.D. Best, M.J. Birnbaum, M.J. Charron, Y.T. Chen, H. Doege, D.E. James, H.F. Lodish, K.H. Moley, J.F. Moley, M. Mueckler, S. Rogers, A. Schurmann, S. Sei- no, B. Thorens (2002) Nomenclature of the GLUT/SLC2A family of sugar/polyol transport facilitators. Am J Physiol Endocrinol Metab 282: E974-E976.

Le Maitre, C.L., P. Baird, A.J. Freemont, J.A. Hoyland (2009) An in vitro study investigating the survival and phenotype of mesenchymal stem cells following injection into nucleus pulposus tissue. Arthritis Res Ther 11: R20.

Le Maitre, C.L., A. Pockert, D.J. Buttle, A.J. Freemont, J.A. Hoyland (2007) Matrix synthesis and degradation in human intervertebral disc degeneration. Biochem Soc Trans 35: 652-655.

Li, Y., S.R. Tew, A.M. Russell, K.R. Gonzalez, T.E. Hardingham, R.E. Hawkins (2004) Transduction of passaged human articular chondrocytes with adenoviral, retroviral, and lentiviral vectors and the effects of enhanced expression of SOX9. Tissue Eng 10: 575-584.

Liu, L.T., B. Huang, C.Q. Li, Y. Zhuang, J. Wang, Y. Zhou (2011) Characteristics of stem cells derived from the degenerated human intervertebral disc cartilage endplate. PLoS One 6: e26285.

Lu, Z.F., B.Z. Doulabi, P.I. Wuisman, R.A. Bank, M.N. Helder (2008) Influence of collagen type II and nucleus pulposus cells on aggregation and differentiation of adipose tissue-derived stem cells. J Cell Mol Med 12: 2812-2822.

Malladi, P., Y. Xu, M. Chiou, A.J. Giaccia, M.T. Longaker (2006) Effect of reduced oxygen tension on chondrogenesis and osteogenesis in adipose-derived mesenchymal cells. Am J Physiol Cell Physiol 290: C1139-C1146.

Mark Erwin, W., D. Islam, E. Eftekarpour, R.D. Inman, M.Z. Karim, M.G. Fehlings (2013) Intervertebral disc-derived stem cells: implications for regenerative medicine and neural repair. Spine (Phila Pa 1976) 38: 211216.

Moore, K.A., I.R. Lemischka (2006) Stem cells and their niches. Science 311: 1880-1885. 
Mwale, F., I. Ciobanu, D. Giannitsios, P. Roughley, T. Steffen, J. Antoniou (2011) Effect of oxygen levels on proteoglycan synthesis by intervertebral disc cells. Spine (Phila Pa 1976) 36: E131-E138.

Risbud, M.V., T.J. Albert, A. Guttapalli, E.J. Vresilovic, A.S. Hillibrand, A.R. Vaccaro, I.M. Shapiro (2004) Differentiation of mesenchymal stem cells towards a nucleus pulposuslike phenotype in vitro: implications for cellbased transplantation therapy. Spine (Phila Pa 1976) 29: 2627-2632.

Roughley, P.J. (2004) Biology of intervertebral disc aging and degeneration: involvement of the extracellular matrix. Spine (Phila Pa 1976) 29: 2691-2699.

Rudert, M., B. Tillmann (1993) Lymph and blood supply of the human intervertebral disc. Cadaver study of correlations to discitis. Acta Orthop Scand 64: 37-40.

Sakai, D. (2008) Future perspectives of cell-based therapy for intervertebral disc disease. Eur Spine J 17: 452-458.

Sakai, D., J. Mochida, T. Iwashina, A. Hiyama, H. Omi, M. Imai, T. Nakai, K. Ando, T. Hotta (2006) Regenerative effects of transplanting mesenchymal stem cells embedded in atelocollagen to the degenerated intervertebral disc. Biomaterials 27: 335-345.
Semenza, G.L. (2000) HIF-1 and human disease: one highly involved factor. Genes Dev 14: 1983-1991.

Semenza, G.L. (2012) Hypoxia-inducible factors in physiology and medicine. Cell 148: 399 408.

Steck, E., H. Bertram, R. Abel, B. Chen, A. Winter, W. Richter (2005) Induction of intervertebral disc-like cells from adult mesenchymal stem cells. Stem Cells 23: 403-411.

Stubbs, S.L., S.T. Hsiao, H.M. Peshavariya, S.Y. Lim, G.J. Dusting, R.J. Dilley (2012) Hypoxic preconditioning enhances survival of human adipose-derived stem cells and conditions endothelial cells in vitro. Stem Cells Dev 21: 1887-1896.

Tapp, H., R. Deepe, J.A. Ingram, M. Kuremsky, E.N. Hanley, Jr., H.E. Gruber (2008) Adiposederived mesenchymal stem cells from the sand rat: transforming growth factor beta and 3D co-culture with human disc cells stimulate proteoglycan and collagen type I rich extracellular matrix. Arthritis Res Ther 10: R89.
Wang, D.W., B. Fermor, J.M. Gimble, H.A. Awad, F. Guilak (2005) Influence of oxygen on the proliferation and metabolism of adipose derived adult stem cells. J Cell Physiol 204: 184191.

Wang, L., R.R. Rao, J.P. Stegemann (2013) Delivery of mesenchymal stem cells in chitosan/ collagen microbeads for orthopedic tissue repair. Cells Tissues Organs 197: 333-343.

Wang, Y.T., X.T. Wu, F. Wang (2010) Regeneration potential and mechanism of bone marrow mesenchymal stem cell transplantation for treating intervertebral disc degeneration. J Orthop Sci 15: 707-719.

Xue, K., L. Qi, G. Zhou, K. Liu (2013) A two-step method of constructing mature cartilage using bone marrow-derived mesenchymal stem cells. Cells Tissues Organs 197: 484-495.

$\checkmark$ Yang, F., V.Y. Leung, K.D. Luk, D. Chan, K.M. Cheung (2009) Mesenchymal stem cells arrest intervertebral disc degeneration through chondrocytic differentiation and stimulation of endogenous cells. Mol Ther 17: 1959-1966.

Zhang, Y.G., X. Guo, P. Xu, L.L. Kang, J. Li (2005) Bone mesenchymal stem cells transplanted into rabbit intervertebral discs can increase proteoglycans. Clin Orthop Relat Res 430: 219-226. 